\title{
Treatment of alcohol dependence with low-dose topiramate: an open-label controlled study
}

\author{
Thomas Paparrigopoulos ${ }^{1 *}$, Elias Tzavellas ${ }^{1 \dagger}$, Dimitris Karaiskos ${ }^{1 \dagger}$, Georgia Kourlaba $^{2 \dagger}$, Ioannis Liappas ${ }^{1 \dagger}$
}

\begin{abstract}
Background: GABAergic anticonvulsants have been recommended for the treatment of alcohol dependence and the prevention of relapse. Several studies have demonstrated topiramate's efficacy in improving drinking behaviour and maintaining abstinence. The objective of the present open-label controlled study was to assess efficacy and tolerability of low-dose topiramate as adjunctive treatment in alcohol dependence during the immediate postdetoxification period and during a 16-week follow-up period after alcohol withdrawal.

Methods: Following a 7-10 day inpatient alcohol detoxification protocol, 90 patients were assigned to receive either topiramate (up to $75 \mathrm{mg}$ per day) in addition to psychotherapeutic treatment ( $\mathrm{n}=30$ ) or psychotherapy alone $(n=60)$. Symptoms of depression and anxiety, as well as craving, were monitored for 4-6 weeks immediately following detoxification on an inpatient basis. Thereafter, both groups were followed as outpatients at a weekly basis for another 4 months in order to monitor their course and abstinence from alcohol.

Results: A marked improvement in depressive $(p<0.01)$, anxiety $(p<0.01)$, and obsessive-compulsive drinking symptoms ( $p<0.01$ ) was observed over the consecutive assessments in both study groups. However, individuals on topiramate fared better than controls $(p<0.01)$ during inpatient treatment. Moreover, during the 4-month follow up period, relapse rate was lower among patients who received topiramate (66.7\%) compared to those who received no adjunctive treatment (85.5\%), $(p=0.043)$. Time to relapse in the topiramate augmentation group was significantly longer compared to the control group (log rank test, $p=0.008$ ). Thus, median duration of abstinence was 4 weeks for the non-medicated group whereas it reached 10 weeks for the topiramate group. No serious side effects of topiramate were recorded throughout the study.

Conclusions: Low-dose topiramate as an adjunct to psychotherapeutic treatment is well tolerated and effective in reducing alcohol craving, as well as symptoms of depression and anxiety, present during the early phase of alcohol withdrawal. Furthermore, topiramate considerably helps to abstain from drinking during the first 16-week postdetoxification period.
\end{abstract}

\section{Background}

Alcohol dependence is a multifactorial disorder influenced by interacting genetic, biological, psychological and environmental factors. Pharmacologically, alcohol is considered to be a potent central nervous system depressant and its action is mediated through multiple neurotransmitter systems, including the GABAergic, glutamatergic, dopaminergic serotoninergic, and opiatergic system. This complex neurobiological network, which is

\footnotetext{
* Correspondence: tpaparrig@med.uoa.gr

+ Contributed equally

'Athens University Medical School, 1st Department of Psychiatry, Eginition

Hospital, Athens, Greece

Full list of author information is available at the end of the article
}

involved in the regulation of alcohol preference, intake, and the rewarding and craving components of alcohol dependence, has been the target of various pharmacological agents, albeit with only limited success. In this context, there has been a growing interest in the use of anticonvulsant medications in the field because these agents may act on the neurobiological substrate of addiction $[1,2]$. In this vein, the anticonvulsant topiramate has been suggested to be promising for treating alcohol dependence $[3,4]$. Although the mechanism is unclear, modulation of the dopamine reward pathways of the brain through antagonizing excitatory glutamate receptors at a-amino-3-hydroxy-5-methylisoxazole-4propionic acid and kainate receptors and inhibiting

\section{Ciomed Central}


dopamine release $[5,6]$ within the mesocorticolimbic system while enhancing inhibitory GABA (by binding to a site of the GABA-A receptor) [7], has been proposed as being responsible for its effectiveness [8]. This dual action of topiramate is supposed first to lead to a dopamine decrease in the nucleus accumbens in response to alcohol ingestion, and consequently to a reduction of its rewarding/reinforcing potential, and second to minimize withdrawal symptoms by moderating the effect of chronic alcohol consumption on neural system excitability. Thus, targeting at both facets of addiction, i.e., craving and feelings of inner tension and discomfort, relapse may be less likely. However, several issues regarding dosing, duration and tolerability of treatment with topiramate have not been adequately addressed as yet.

Treatment of alcohol dependence is a two-phase process, which aims at alcohol withdrawal and subsequent long-term abstinence and relapse prevention [9]. Depending on the phase, different priorities may be set. Thus, pharmacotherapy during and immediately after detoxification may protect from withdrawal dysphoric symptoms and can reduce anxiety and symptoms of depression [10-12]. Thereafter, medication can be used to reduce craving and reward from alcohol use. GABAergic and glutamatergic medications might be promising candidates for helping during both phases of alcohol dependence treatment [13].

The objective of the present open-label controlled study was to assess the efficacy and tolerability profile of lowdose topiramate as adjunctive treatment in alcohol dependence during the immediate post-detoxification period and during a 16-week follow-up period after alcohol withdrawal. The choice of low-dose topiramate was made based on the existing, albeit limited, literature, which suggests that even low doses of this medication can be beneficial in preventing alcohol relapse [14,15,4]; moreover, a less aggressive approach with milder side-effects could be advantageous in terms of treatment adherence. A control non-medicated group of alcohol-dependent individuals was used for comparisons in terms of anxiety and depressive symptoms, craving and drinking outcome.

\section{Methods}

\section{Study design - participants}

The study was an open-label controlled clinical trial. Participants were assigned either to a standard alcohol detoxification group (see below) or to a topiramate augmentation group. Assignment to the topiramate augmentation group was made on a 2:1 ratio; thus, every third intake was assigned to the topiramate group. In total, 90 alcohol-dependent individuals who consecutively contacted the Drug and Alcohol Addiction Clinic of the Athens University Psychiatric Clinic at the Eginition Hospital in Athens, Greece, were enrolled in the study.
Patients had to fulfil the DSM-IV-TR [16] diagnostic criteria for alcohol abuse/dependence and were admitted for inpatient alcohol detoxification. Informed consent was obtained from the participants after providing detailed information on the objectives of the study and the research/therapeutic protocol. All procedures were approved by the ethical committee of our institution. ("Committee on Medical Ethics of the Eginition Hospital" \& Reference No: 1178).

The inclusion criteria were: a) age 18-65 years, b) absence of a serious physical illness (as assessed through physical examination and routine laboratory screening), c) absence of another pre- or co-existing major psychiatric disorder on the DSM-IV-TR axis I, d) absence of another drug abuse, and e) participants with affective or anxiety symptoms were not excluded from the study if concurrent with an alcohol-abusing period; individuals who fulfilled a DSM-IV-TR diagnosis of depressive or anxiety disorder (assessed through the Schedules for Clinical Assessment in Neuropsychiatry [SCAN] [17] and information obtained from a close relative) were excluded from the study if relevant criteria were met prior to the onset of alcoholism or during periods of abstinence.

Participants were assigned to two study groups: the control group $(n=60)$, which included subjects treated with a standard alcohol detoxification protocol, and the topiramate augmentation group $(\mathrm{n}=30)$, which included patients who were additionally given topiramate (up to $75 \mathrm{mg} /$ day in 2 divided doses). Topiramate was initiated at a daily dose of $25 \mathrm{mg}$, before stopping the last dose of $5 \mathrm{mg}$ diazepam, and was gradually increased up to $75 \mathrm{mg} /$ day over three weeks (mean dose: $55.0 \pm 19.03 \mathrm{mg} /$ day).

The standard alcohol detoxification protocol was initiated and completed one week (7-10 days) after admission to the ward. This protocol includes vitamin replacement (vitamins $\mathrm{C}, \mathrm{E}$ and $\mathrm{B}$ complex) and oral administration of diazepam (30-60 mg in divided doses), with gradual taper off over a week. Thereafter, both groups were given a standard treatment program with cognitive-behavioural short-term psychotherapy of 46 week duration (i.e. during their inpatient treatment). After discharge, patients were assessed at a weekly basis for 4 more months in order to monitor their course and abstinence from alcohol. Assessment of abstinence from alcohol was based on self reports, but it was further cross-checked with a family member to ascertain accuracy of information. Also, serum $\gamma$-glutamyl transpeptidase $(\gamma-\mathrm{GT})$ and an alcohol breath test at each visit were used to control abstinence. No discrepancies were observed between these measures of abstinence throughout the study. Although there is ongoing debate regarding the reliability of self-reports of alcohol consumption, 
it has been shown that data thus collected are a valid source of information in the case of dependent individuals [18]. Furthermore, $\gamma$-GT is considered to be a reliable marker of alcohol relapse detection [19].

From the total sample $(\mathrm{n}=90)$, eighty-five subjects $(\mathrm{n}=85)$ were included in the final statistical analysis because five participants from the control group had occasionally used benzodiazepines during the follow-up period on their own initiative, and this was considered as protocol violation.

\section{Measures}

Participants were diagnosed by the Schedules for Clinical Assessment in Neuropsychiatry [SCAN] and assessed through the Composite International Diagnostic Interview [20] (CIDI; section on alcohol consumption) for their pattern of alcohol abuse, potential major life problems related to alcohol consumption and the occurrence of withdrawal symptoms in the past. All data pertaining to alcohol use were self-reported but in order to ascertain accuracy of information a relative was also interviewed to corroborate current status and psychiatric history. Furthermore, sociodemographic data (age, socioeconomic status, marital status, level of education) and previous psychiatric history (pre-existent diagnosis, medication, number of hospitalizations) were recorded. Symptoms of depression and anxiety were assessed with the Hamilton Depression Rating Scale (HDRS) [21] and the Hamilton Anxiety Rating Scale (HARS) [22]. Obsessive thoughts about alcohol use and compulsive behaviours toward drinking (facets of craving) were estimated with the Obsessive Compulsive Drinking Scale (OCDS) [23]. Overall functioning was assessed using the Global Assessment Scale (GAS) [24]. The severity of withdrawal symptoms was evaluated twice daily during the first week of alcohol withdrawal with a modified version of the Addiction Research Foundation Clinical Institute Withdrawal Assessment for Alcohol (CIWA-Ar) [25]. Adverse effects of treatment in both groups were monitored through an adapted version of the Systematic Assessment for Treatment Emergent Events (COMBINE SAFTEE) scale, which is a structured instrument for collecting adverse events adapted for clinical studies in the alcoholism field [26]. Assessments were done at three time points; initially within $48 \mathrm{~h}$ upon entering the program (time point 0 ) and subsequently at $21 \pm 2$ day intervals (time point $1 \& 2$ ) over the 4-6 week study period.

\section{Statistical analysis}

The number of subjects that entered into the final analysis was eighty-five $(n=85)$. Independent samples $t$-tests were used to evaluate differences between groups in terms of symptoms of depression, anxiety, global functioning and obsessive-compulsive drinking scores at the different time points. Within groups differences were estimated with repeated measures analysis of variance (RMANOVA). For all reported values the means $( \pm \mathrm{SD})$ were calculated. Chisquare statistics were used to compare categorical variables, as appropriate. Cox proportional hazards model was used to estimate the hazard ratio (HR) of achieving 16 weeks of continuous abstinence and the Kaplan-Meier method was applied to calculate the cumulative probability function of reaching 16 weeks of abstinence for the topiramate and the control group. The log-rank test was used to compare the cumulative probability functions. The proportional hazard assumption of Cox model was assessed through the appropriate graph. All tests were two-tailed with statistical significance set at $\mathrm{p}<0.05$. Data analysis was performed using the SPSS statistical software package (SPSS Inc. Chicago, IL, USA).

\section{Results}

No significant differences were observed between the control and topiramate group in terms of their sociodemographic characteristics, as well as the variables related to alcohol abuse history and withdrawal symptoms during the first week of abstinence (Table 1). As regards psychopathological symptoms both groups had similarly high scores on the HDRS, HARS and OCDS, and low GAS scores upon admission (time 0), which represent a serious psychosocial impairment. A marked improvement on all these measures was observed in the two subsequent assessments (time 1 \& time 2 ) in both study groups [depression $(\mathrm{p}<0.01)$, anxiety $(\mathrm{p}<0.01)$, and obsessive-compulsive drinking symptoms $(\mathrm{p}<0.01)$ ]. However, subjects on topiramate did significantly better than controls concerning mood improvement, i.e., anxiety and depression $(\mathrm{p}<0.05)$, and craving as well $(\mathrm{p}<$ 0.01) (Table 2).

Table 1 Sociodemographic characteristics and variables related to alcohol consumption of the sample $(\mathbf{N}=\mathbf{8 5})$

\begin{tabular}{|c|c|c|}
\hline & $\begin{array}{c}\text { Topiramate } \\
\text { group } \\
(\mathbf{N}=30)\end{array}$ & $\begin{array}{l}\text { Control group* } \\
\qquad(\mathrm{N}=55)\end{array}$ \\
\hline Mean age, years $( \pm S D)$ & $43.8 \pm 8.1$ & $46.3 \pm 11.0$ \\
\hline $\operatorname{Sex}(M, F)$ & $M: 27, F: 3$ & $M: 48, F: 7$ \\
\hline Family status (S, M, D) & S: $8, M: 16, D: 6$ & S: $10, \mathrm{M}: 33, \mathrm{D}: 12$ \\
\hline Socioeconomic status $(H, M, L)$ & $\mathrm{H}: 1, \mathrm{M}: 25, \mathrm{~L}: 4$ & $\mathrm{H}: 2, \mathrm{M}: 39, \mathrm{~L}: 14$ \\
\hline Educational years & $7.6 \pm 3.1$ & $8.3 \pm 3.8$ \\
\hline Age at onset, years ( \pm SD) & $24.1 \pm 6.2$ & $27.3 \pm 9.6$ \\
\hline $\begin{array}{l}\text { Mean alcohol consumption } \\
\text { (gr/day) }\end{array}$ & $272 \pm 115$ & $284 \pm 140$ \\
\hline $\begin{array}{l}\text { Mean (CIWA-Ar) during the first } \\
\text { week }\end{array}$ & $26.1 \pm 6.7$ & $25.7 \pm 6.3$ \\
\hline
\end{tabular}

M, Male; F, Female; S, Single; M, Married; D, Divorced/separated/widowed; $H$, High; M, Middle; L, Low. CIWA-Ar, Clinical Institute Withdrawal Assessment for Alcohol, Revised.

${ }^{*}$ All comparisons between groups were non-significant. 
Table 2 Mean scores \pm SD of the various measures of psychopathology and craving at the different time points of assessment (time $\mathbf{0} \rightarrow$ time 2 ) in the control and the topiramate augmentation group

\begin{tabular}{|c|c|c|c|c|}
\hline $\begin{array}{c}\text { Variable } \\
(\text { Mean } \pm \text { SD) }\end{array}$ & Group & $1^{\text {st }}$ Assessment (time 0 ) & $\begin{array}{l}2^{\text {nd }} \text { Assessment } \\
\text { (time 1) }\end{array}$ & $\begin{array}{c}3^{\text {rd }} \text { Assessment } \\
\text { (time 2) }\end{array}$ \\
\hline \multicolumn{5}{|l|}{ HDRS } \\
\hline & Controls & $38.7 \pm 7.6$ & $15.9 \pm 8.6+$ & $8.1 \pm 6.6 \infty$ \\
\hline & Topiramate & $39.6 \pm 5.5$ & $12.5 \pm 3.1^{*}+$ & $4.9 \pm 3.1^{*} \infty$ \\
\hline \multicolumn{5}{|l|}{ HARS } \\
\hline & Controls & $30.5 \pm 10.2$ & $13.9 \pm 6.7+$ & $7.1 \pm 6.2 \infty$ \\
\hline & Topiramate & $31.8 \pm 5.3$ & $10.9 \pm 3.6^{*}+$ & $4.3 \pm 3.8^{*} \infty$ \\
\hline \multicolumn{5}{|l|}{ GAS } \\
\hline & Controls & $46.7 \pm 5.1$ & $75.6 \pm 9.3+$ & $85.4 \pm 8.5 \infty$ \\
\hline & Topiramate & $46.6 \pm 4.7$ & $74.3 \pm 6.7+$ & $84.3 \pm 5.6 \infty$ \\
\hline \multicolumn{5}{|l|}{ OCDS } \\
\hline & Controls & $37.2 \pm 8.3$ & $17.3 \pm 3.9+$ & $13.3 \pm 2.8 \infty$ \\
\hline & Topiramate & $37.6 \pm 7.8$ & $15.3 \pm 3.9^{*}+$ & $10.3 \pm 3.1^{* *} \infty$ \\
\hline
\end{tabular}

Statistics: Between groups (independent samples t-test); within groups (RMANOVA).

*Significant difference between controls and topiramate augmentation group $(<0.05)$.

** Significant difference between controls and topiramate augmentation group $(<0.01)$.

+ Significant difference between $2^{\text {nd }}$ and $1^{\text {st }}$ assessment $(<0.01)$.

$\infty$ Significant difference between 3rd and 2nd assessment $(<0.01)$.

Long-term outcome in terms of abstinence from alcohol was better for the topiramate augmentation group. Thus, although 67 patients in total $(78.8 \%)$ had relapsed to alcohol use by the end of the study (16 weeks after discharge), relapse rate was significantly lower in the topiramate group (66.7\%) compared with the control group $(85.5 \%)(p=0.043)$. Also, median duration of abstinence in the topiramate group was significantly longer compared to the non-medicated group (10 weeks vs. 4 weeks; log rank test, $\mathrm{p}=0.008$, Figure 1$)$. Cox proportional hazard model showed that risk of relapse was $56 \%$ lower among patients receiving topiramate compared to controls $(\mathrm{HR}=0.515,95 \% \mathrm{CI}: 0.304-0.874, \mathrm{p}=0.014)$.

Reported adverse effects are presented on Table 3. A considerable proportion (> 10\%) of the topiramate augmentation group had some adverse effects, but no significant difference was recorded compared with the control group, except for somnolence which was significantly more frequent in the topiramate group ( $23.3 \%$ vs. $5.4 \%)$. All adverse effects were tolerable and there were no dropouts from the study.

\section{Discussion}

The main finding of the present study is that low-dose topiramate given as a treatment adjunct is well-accepted and effective in reducing craving for alcohol and symptoms of anxiety and depression during the early phase of alcohol withdrawal. Furthermore, topiramate combined with a psychotherapeutic intervention improves abstinence from drinking during the first 16-week postdetoxification period, in comparison with alcohol-dependent individuals receiving psychotherapy alone.
Although topiramate is not currently approved for the treatment of alcohol dependence [27], several randomized double-blind placebo-controlled trials have demonstrated its efficacy in improving drinking behaviour and maintaining abstinence [28-30]. Compared to the standard medications approved for alcohol dependence, topiramate has been found to be inferior to disulfiram in terms of days to relapse [31] and superior to naltrexone in reducing craving [32] and improving some other critical measures of drinking behaviour [30]. The




Table 3 Reported adverse effects by study group

\begin{tabular}{lcc}
\hline & $\begin{array}{c}\text { Topiramate group } \\
\mathbf{N}=\mathbf{3 0}(\%)\end{array}$ & $\begin{array}{c}\text { Control group } \\
\mathbf{N}=\mathbf{5 5}(\%)\end{array}$ \\
\hline Dizziness & $6(20.0)$ & $4(7.2)$ \\
\hline Somnolence & $7(23.3)$ & $3(5.4)^{*}$ \\
\hline Nervousness & $2(6.6)$ & $7(12.7)$ \\
\hline Numbness/Paresthesias & $3(10.0)$ & $3(5.4)$ \\
\hline Psychomotor slowness & $4(13.3)$ & $2(3.6)$ \\
\hline Nausea & $5(16.6)$ & $2(3.6)$
\end{tabular}

* Fisher's Exact Test (2-tailed sig.), $\mathrm{p}<0.05$.

precise mechanism of topiramate's favourable action is unclear. It may be that it modulates GABAergic transmission in the central amygdala, a brain region implicated in the regulation of emotionality and alcohol intake $[33,34]$. Also, it has been shown that GABA receptors undergo allosteric modulation by ethanol and mediate the acute and chronic effects of alcohol, including tolerance, dependence and withdrawal [35]. On the other hand, topiramate enhances the inhibitory function of GABA, antagonizes excitatory glutamate receptors, and inhibits dopamine release [36].

Topiramate has been used for the treatment of alcohol dependence in outpatient settings. Doses ranging from 150 to $300 \mathrm{mg} /$ day have shown promising results, in terms of significant improvement in several dependence-related parameters [3]. However, a major concern has been topiramate's adverse effects, which are prominent especially during the titration period, appear to be dose-related but usually subside with continued treatment $[37,38]$. Thus, the majority of patients who discontinue topiramate treatment, due to its side effects, do so early in treatment. In this line of thought, a key objective of the present study was to establish the efficacy and side effect profile of lowdose topiramate (up to $75 \mathrm{mg} /$ day) that might improve adherence to treatment. In our sample, a considerable proportion (>10\%) of the topiramate augmentation group had some adverse effects, but no significant difference was recorded compared with the control group. However, these adverse effects were tolerable and there were no dropouts from the study. This was probably due to several reasons such as that the initial detoxification took place in an inpatient basis assuring a high compliance with all treatment interventions, that the study population was highly motivated to withdraw from alcohol, and finally that our sample consisted of individuals with relatively high initial withdrawal symptoms who are usually excluded from most studies of outpatient populations.

Impulsive and compulsive behaviours play a crucial role in alcohol abuse, craving and relapse [39-41]; therefore, medications with anticraving properties have been used for prevention of relapse. Several studies have demonstrated topiramate's efficacy in the management of impulsive, aggressive and self-harmful behaviour [42], gambling [43], eating disorders [44], as well as an adjunct to SSRIs in obsessive-compulsive disorder [45]. Thus, moderation of impulsivity with consequent minimization of craving might be responsible for the lower rates of relapse in the topiramate augmentation group. Moreover, research has consistently documented a strong association between anxiety and/or symptoms of depression and alcohol abuse; these symptoms usually subside following a few weeks of abstinence [46]. However, mild anxiety [11] and minor symptoms of depression may persist for several months and various medicines - including tiagabine [47], mirtazapine and venlafaxine [12] - have been used adjunctively to standard alcohol detoxification treatment in order to increase patient compliance and improve treatment outcome $[10,48]$. Through such a collateral beneficial action, topiramate could lead to the more favourable outcome observed in the augmentation group of the present study.

The main limitations of the present study are: a) the relatively small sample size, which reduces the statistical significance of our findings, b) the study did not follow a double-blind placebo control design; such a design was not feasible due to the ethical restrictions of our institution, c) assessment of alcohol use during the follow-up period was mostly based on self-reports and periodically cross-checked with an informant and $\gamma$-GT measurements, and d) a longer follow-up period would provide important information on the long term efficacy of topiramate in a community setting. Despite the above limitations, our results corroborate previous reports that show the potential usefulness of topiramate in the treatment of alcohol dependence even when administered at low doses.

\section{Conclusions}

In conclusion, low-dose topiramate when used as an adjunct to psychotherapy is well tolerated and effective in reducing alcohol craving, as well as symptoms of depression and anxiety, present during the early phase of alcohol withdrawal. Furthermore, topiramate considerably helps to abstain from drinking during the first 16 -week post-detoxification period, a period which is critical for relapse. Thus, topiramate could be an alternative option beyond the already approved agents for the treatment of alcohol dependence.

\section{Abbreviations}

GABA: $\gamma$-Aminobutyric acid; DSM-IV-TR: Diagnostic and Statistical Manual of Mental Disorders, Fourth Edition, Text Revision; SCAN: Schedules for Clinical Assessment in Neuropsychiatry; $\gamma$-GT: $\gamma$-glutamyl transpeptidase; CIDI: Composite International Diagnostic Interview; HDRS: Hamilton Depression Rating Scale; HARS: Hamilton Anxiety Rating Scale; OCDS: Obsessive Compulsive Drinking Scale; GAS: Global Assessment Scale; CIWA-Ar: Clinical Institute Withdrawal Assessment for Alcohol, revised; SAFTEE: Systematic Assessment for Treatment Emergent Events; RMANOVA: repeated measures 
analysis of variance; SD: standard deviation; HR: hazard ratio; SSRI: Selective serotonin reuptake inhibitor

\section{Acknowledgements}

The authors wish to thank the nursing personnel of the Palaiologou Ward at the Eginition Hospital, as well as Mrs Urania Savva for their assistance in conducting this research.

\section{Author details}

'Athens University Medical School, 1st Department of Psychiatry, Eginition Hospital, Athens, Greece. ${ }^{2}$ Harokopio University of Athens, Department of Nutrition and Dietetics, Athens, Greece.

\section{Authors' contributions}

TP participated in the design of the study and drafted the manuscript; ET participated in the design of the study, data collection, and assisted in drafting the manuscript; DK participated in data collection, in the analysis and interpretation of data, and helped to draft the manuscript; IL had overall supervision of the study and made extensive revisions of the manuscript; GK participated in data analysis and their interpretation. All authors read and approved the final manuscript.

\section{Competing interests}

The authors declare that they have no competing interests.

Received: 21 November 2010 Accepted: 14 March 2011

Published: 14 March 2011

\section{References}

1. Johnson BA, Swift RM, Ait-Daoud N, DiClemente CC, Javors MA, Malcolm RJ $\mathrm{Jr}$ : Development of novel pharmacotherapies for the treatment of alcohol dependence: focus on antiepileptics. Alcohol Clin Exp Res 2004, 28(2):295-301.

2. Lobo IA, Harris RA: GABA(A) receptors and alcohol. Pharmacol Biochem Behav 2008, 90(1):90-94.

3. Arbaizar B, Diersen-Sotos T, Gomez-Acebo I, Llorca J: Topiramate in the treatment of alcohol dependence: a meta-analysis. Actas Esp Psiquiatr 2010, 38(1):8-12

4. Johnson BA, Ait-Daoud N: Topiramate in the new generation of drugs: efficacy in the treatment of alcoholic patients. Curr Pharm Des 2010, 16(19):2103-2112.

5. Deng C, Li KY, Zhou C, Ye JH: Ethanol enhances glutamate transmission by retrograde dopamine signaling in a postsynaptic neuron/synaptic bouton preparation from the ventral tegmental area. Neuropsychopharmacology 2009, 34(5):1233-1244.

6. Xiao C, Shao XM, Olive MF, Griffin WC, Li KY, Krnjevic K, Zhou C, Ye JH: Ethanol facilitates glutamatergic transmission to dopamine neurons in the ventral tegmental area. Neuropsychopharmacology 2009, 34(2):307-318.

7. Heilig M, Egli M: Pharmacological treatment of alcohol dependence: target symptoms and target mechanisms. Pharmacol Ther 2006, 111(3):855-876.

8. Johnson BA: Progress in the development of topiramate for treating alcohol dependence: from a hypothesis to a proof-of-concept study. Alcohol Clin Exp Res 2004, 28(8):1137-1144

9. Kleber HD, Weiss RD, Anton RF, George TP, Greenfield SF, Kosten TR, O'Brien CP, Rounsaville BJ, Strain EC, Ziedonis DM, et al: Treatment of patients with substance use disorders, second edition. American Psychiatric Association. Am J Psychiatry 2007, 164(4 Suppl):5-123.

10. Driessen $M$, Meier $S$, Hill A, Wetterling T, Lange $W$, Junghanns $K$ : The course of anxiety, depression and drinking behaviours after completed detoxification in alcoholics with and without comorbid anxiety and depressive disorders. Alcohol Alcohol 2001, 36(3):249-255.

11. Willinger $U$, Lenzinger $E$, Hornik $K$, Fischer $G$, Schonbeck G, Aschauer HN Meszaros $K$ : Anxiety as a predictor of relapse in detoxified alcoholdependent patients. Alcohol Alcohol 2002, 37(6):609-612.

12. Liappas J, Paparrigopoulos T, Tzavellas E, Rabavilas A: Mirtazapine and venlafaxine in the management of collateral psychopathology during alcohol detoxification. Prog Neuropsychopharmacol Biol Psychiatry 2005, 29(1):55-60.

13. Vengeliene V, Bilbao A, Molander A, Spanagel R: Neuropharmacology of alcohol addiction. Br J Pharmacol 2008, 154(2):299-315.
14. Chiu YH, Lee TH, Shen WW: Use of low-dose topiramate in substance use disorder and bodyweight control. Psychiatry Clin Neurosci 2007, 61(6):630-633.

15. Martinotti G, Di Nicolaa M, De Vitaa O, Tedeschia D, Guerrieroa L, Guglielmoa R, De Filippisa R, Janiri L: Low-dosage topiramate in alcohol dependence: a randomized, double-blind, placebo-controlled trial. European Psychiatry, 18th European Congress of Psychiatry, Poster: 2010 2010, 1665.

16. Diagnostic and statistical manual of mental disorders DSM-IV-TR (Revised 4th ed). Washington, DC: American Psychiatric Association; 2000.

17. Wing JK, Babor T, Brugha T, Burke J, Cooper JE, Giel R, Jablenski A, Regier D, Sartorius N: SCAN. Schedules for Clinical Assessment in Neuropsychiatry. Arch Gen Psychiatry 1990, 47(6):589-593.

18. Magura S, Kang SY: Validity of self-reported drug use in high risk populations: a meta-analytical review. Subst Use Misuse 1996, 31(9):1131-1153.

19. Salaspuro M: Carbohydrate-deficient transferrin as compared to other markers of alcoholism: a systematic review. Alcohol 1999, 19(3):261-271.

20. Robins LN, Wing J, Wittchen HU, Helzer JE, Babor TF, Burke J, Farmer A, Jablenski A, Pickens R, Regier DA, et al: The Composite International Diagnostic Interview. An epidemiologic Instrument suitable for use in conjunction with different diagnostic systems and in different cultures. Arch Gen Psychiatry 1988, 45(12):1069-1077.

21. Hamilton M: A rating scale for depression. I Neurol Neurosurg Psychiatry 1960, 23:56-62.

22. Hamilton $\mathrm{M}$ : The assessment of anxiety states by rating. $\mathrm{Br} J$ Med Psychol 1959, 32(1):50-55.

23. Anton RF, Moak DH, Latham P: The Obsessive Compulsive Drinking Scale: a self-rated instrument for the quantification of thoughts about alcohol and drinking behavior. Alcohol Clin Exp Res 1995, 19(1):92-99.

24. Endicott J, Spitzer RL, Fleiss JL, Cohen J: The global assessment scale. A procedure for measuring overall severity of psychiatric disturbance. Arch Gen Psychiatry 1976, 33(6):766-771.

25. Sullivan JT, Sykora K, Schneiderman J, Naranjo CA, Sellers EM: Assessment of alcohol withdrawal: the revised clinical institute withdrawal assessment for alcohol scale (CIWA-Ar). Br J Addict 1989, 84(11):1353-1357.

26. Johnson BA, Ait-Daoud N, Roache JD: The COMBINE SAFTEE: a structured instrument for collecting adverse events adapted for clinical studies in the alcoholism field. J Stud Alcohol Supp/ 2005, , 15: 157-167, discussion 140.

27. Olive MF: Pharmacotherapies for alcoholism: the old and the new. CNS Neurol Disord Drug Targets 2010, 9(1):2-4.

28. Johnson BA, Ait-Daoud N, Bowden CL, DiClemente CC, Roache JD, Lawson K, Javors MA, Ma JZ: Oral topiramate for treatment of alcohol dependence: a randomised controlled trial. Lancet 2003, 361(9370):1677-1685.

29. Johnson BA, Rosenthal N, Capece JA, Wiegand F, Mao L, Beyers K, McKay A, Ait-Daoud N, Anton RF, Ciraulo DA, et al: Topiramate for treating alcohol dependence: a randomized controlled trial. Jama 2007, 298(14):1641-1651.

30. Baltieri DA, Daro FR, Ribeiro PL, de Andrade AG: Comparing topiramate with naltrexone in the treatment of alcohol dependence. Addiction 2008, 103(12):2035-2044.

31. De Sousa AA, De Sousa J, Kapoor H: An open randomized trial comparing disulfiram and topiramate in the treatment of alcohol dependence. J Subst Abuse Treat 2008, 34(4):460-463.

32. Florez G, Saiz PA, Garcia-Portilla P, Alvarez S, Nogueiras L, Bobes J: Topiramate for the Treatment of Alcohol Dependence: Comparison with Naltrexone. Eur Addict Res 2010, 17(1):29-36.

33. Gass JT, Olive MF: Glutamatergic substrates of drug addiction and alcoholism. Biochem Pharmacol 2008, 75(1):218-265.

34. Roberto M, Madamba SG, Moore SD, Tallent MK, Siggins GR: Ethanol increases GABAergic transmission at both pre- and postsynaptic sites in rat central amygdala neurons. Proc Natl Acad Sci USA 2003, 100(4):2053-2058.

35. Enoch MA: The role of GABA(A) receptors in the development of alcoholism. Pharmacol Biochem Behav 2008, 90(1):95-104.

36. Olmsted CL, Kockler DR: Topiramate for alcohol dependence. Ann Pharmacother 2008, 42(10):1475-1480.

37. Luykx JJ, Carpay JA: Nervous system adverse responses to topiramate in the treatment of neuropsychiatric disorders. Expert Opin Drug Saf 2010, 9(4):623-631. 
38. Meador KJ: Cognitive effects of levetiracetam versus topiramate. Epilepsy Curr 2008, 8(3):64-65.

39. Charney DA, Zikos E, Gill KJ: Early recovery from alcohol dependence: factors that promote or impede abstinence. I Subst Abuse Treat 2010, 38(1):42-50.

40. Dawe S, Loxton NJ: The role of impulsivity in the development of substance use and eating disorders. Neurosci Biobehav Rev 2004, 28(3):343-351

41. Koob GF: Animal models of craving for ethanol. Addiction 2000, 95(Suppl 2):S73-81.

42. Prado-Lima PA: [Pharmacological treatment of impulsivity and aggressive behavior]. Rev Bras Psiquiatr 2009, 31(Suppl 2):S58-65.

43. Leung KS, Cottler LB: Treatment of pathological gambling. Curr Opin Psychiatry 2009, 22(1):69-74

44. McElroy SL, Guerdjikova Al, Martens B, Keck PE, Pope HG, Hudson Jl: Role of antiepileptic drugs in the management of eating disorders. CNS Drugs 2009, 23(2):139-156.

45. Berlin HA, Koran LM, Jenike MA, Shapira NA, Chaplin W, Pallanti S, Hollander E: Double-blind, placebo-controlled trial of topiramate augmentation in treatment-resistant obsessive-compulsive disorder. J Clin Psychiatry 2010

46. Liappas J, Paparrigopoulos T, Tzavellas E, Christodoulou G: Impact of alcohol detoxification on anxiety and depressive symptoms. Drug Alcohol Depend 2002, 68(2):215-220.

47. Paparrigopoulos T, Tzavellas E, Karaiskos D, Malitas P, Liappas I: An open pilot study of tiagabine in alcohol dependence: tolerability and clinical effects. J Psychopharmacol 2010, 24(9):1375-1380.

48. Bottlender M, Soyka M: Impact of craving on alcohol relapse during, and 12 months following, outpatient treatment. Alcohol Alcohol 2004, 39(4):357-361.

\section{Pre-publication history}

The pre-publication history for this paper can be accessed here: http://www.biomedcentral.com/1471-244X/11/41/prepub

doi:10.1186/1471-244X-11-41

Cite this article as: Paparrigopoulos et al: Treatment of alcohol

dependence with low-dose topiramate: an open-label controlled study. BMC Psychiatry 2011 11:41.

\section{Submit your next manuscript to BioMed Central and take full advantage of:}

- Convenient online submission

- Thorough peer review

- No space constraints or color figure charges

- Immediate publication on acceptance

- Inclusion in PubMed, CAS, Scopus and Google Scholar

- Research which is freely available for redistribution

Submit your manuscript at www.biomedcentral.com/submit 